\title{
First trimester combined screening for fetal aneuploidies enhanced with additional ultrasound markers: an 8-year prospective study
}

\author{
Dragos Nemescu ${ }^{\text {, Adina Bratie}}{ }^{1}$, Alexandra Mihaila ${ }^{1}$, Dan Navolan², Adina Tanase ${ }^{1}$ \\ ${ }^{1}$ Grigore T Popa University of Medicine and Pharmacy, Department of Obstetrics and Gynecology, Iasi, Romania \\ ${ }^{2}$ Department of Obstetrics and Gynecology, Victor Babes University of Medicine and Pharmacy, Timisoara, Romania
}

\begin{abstract}
Objectives: To describe our screening population and audit of the performance of first-trimester screening for Down syndrome, based on a combined test, enhanced with additional ultrasound markers, over the whole period of the study.

Material and methods: We performed a prospective study from 2009 to 2016, which included 1358 singleton fetuses with a crown-rump length of $45-84 \mathrm{~mm}$. The risk of aneuploidy was calculated using nuchal translucency, fetal heart rate (FHR), and additional markers, such as nasal bone (NB), tricuspid flow (TF) and ductus venosus (DV), combined with maternal serum free $\beta$-human chorionic gonadotropin ( $f \beta-h C G)$ and pregnancy-associated plasma protein-A (PAPP-A).

Results: $87 \%$ of patients were evaluated using all the additional ultrasound markers and $97 \%$ of patients were assessed using at least two markers, in any combination. $70.5 \%$ of patients were also evaluated using maternal serum biochemistry. The most common risk calculation used nuchal translucency, FHR, all additional ultrasound markers, $\mathrm{f} \beta$-hCG and PAPP-A in 851 (62.7\%) of cases. The adjusted risk of trisomy 21 was greater than 1:100 in 65 (4.8\%) women. Of these patients, 58 (87.7\%) chose to have an invasive test. There were 24 aneuploid fetuses (1.7\%); and from these we identified $12(50 \%)$ trisomy 21 , $6(25 \%)$ sex chromosome anomalies, with the remainder being triploidy and trisomy $18 / 13$. The combined test detected 11 of the 12 cases as having trisomy 21 , with a first trimester detection rate of $91.7 \%$. 39 fetuses (2.8\%) had various types of structural anomalies.
\end{abstract}

Conclusion: The combined test enhanced with all additional ultrasound markers did not show any substantial improvement in T21 detection rate, when compared with using only one of the additional markers.

Key words: first-trimester, screening, nuchal translucency, combined test, trisomy 21

Ginekologia Polska 2018; 89, 4: 205-210

\section{INTRODUCTION}

Screening for chromosomal and structural anomalies at the end of the first trimester of pregnancy has developed rapidly in the last decade and is now important in prenatal consultation. It evolved as a combination of considering maternal age, fetal nuchal translucency (NT) thickness, maternal serum free $\beta$-human chorionic gonadotropin ( $\beta$-hCG) and pregnancy associated plasma protein-A (PAPP-A) at 11-13 weeks' gestation [1]. Usually, the accuracy of the screening is increased by assessing additional ultrasound markers, such as the nasal bone (NB) [2], the tricuspid flow (TR) [3], the ductus venosus flow (DV) [4] and the fetal heart rate (FHR) [5].
The screening algorithm was developed by the Fetal Medicine Foundation (FMF) [6] and is known as the combined first trimester test. It uses an a priori aneuploidy risk derived from maternal age and maternal history, multiplied by likelihood ratios resulting from the deviation of the measured individual values from the respective expected median [7]. It was validated in large prospective studies and its simplest form, combining NT and maternal biochemistry, can detect $90 \%$ of trisomy 21 (T21) affected pregnancies at a false-positive rate (FPR) of about $5 \%[5,8]$. A positive consequence of screening for $T 21$ is the early diagnosis of trisomies 18 and 13 , which are the second and third most common chromosomal abnormalities [9]. 
When the combined screening test is enhanced with one of the additional ultrasound markers such as NB, TR or DV, the T21 detection rate improves to $91-96 \%$, while reducing the FPR to about $2.5 \%[6,10]$. Few studies have examined the screening performance when all additional markers are assessed together. Using an ultrasound only based protocol, with NT and all additional markers, for a FPR of $5 \%$, the detection rate for 721 reached $94-97.5 \%$ [11-13]. When all of these markers were added to a traditional combined test, the detection rate substantially increased to up to $100 \%$, for a FPR of $2.4 \%$ [14] or $3.4 \%$ [15].

The aim of our study was to describe the screening population and to audit the performance of a first-trimester combined test, enhanced with additional ultrasound markers, in 1358 prospectively examined singleton pregnancies. Our hypothesis was that the screening performance would be like that estimated by the original model and observed in other large studies.

\section{MATERIAL AND METHODS}

The data for our study resulted from the clinical implementation of a combined test in screening for aneuploidies at 11 to 13 weeks' gestation in women with singleton pregnancies, with a crown-rump length of $45-84 \mathrm{~mm}$, who attended our Fetal Medicine Centre (private practice), from January 2009 to December 2016. Written informed consent was obtained from each patient and the study followed the principles of the Declaration of Helsinki.

Fetal evaluation was realized prospectively, according to the FMF protocol for combined first trimester test screening for trisomy 21 [8]. The ultrasound scans were done transabdominally (or transvaginal if needed) to estimate the gestational age through the measurement of the fetal crown-rump length (CRL) and to diagnose any major fetal anomalies [16].

NT thickness was measured at the time of the examination, together with an evaluation of all additional (secondary) first trimester ultrasound markers (NB, TR, DV, FHR) if this was technically possible [3]. All scans were performed by FMF certified operators, who passed an annual audit based on the distribution of their NT measurements (www.fetalmedicine.org), using mainly Voluson E8 but also E6 and 730 (GE Medical Systems, Zipf, Austria) ultrasound machines. We followed the ALARA (As Low As Reasonably Achievable) principle for acoustic power and our output data was within the limits already described [17].

Samples of maternal blood for biochemistry screening were collected at 9 to 14 weeks, depending on the time of patients' counselling sessions. Concentrations of PAPP-A and free b-hCG were measured by external laboratories using the Cobas (Roche, Switzerland), Delfia Express (Perkin Elmer, USA), Immulite (Siemens AG, Germany) or Kryptor (Brahms AG, Germany) systems.
Finally, the risks of aneuploidies were calculated using the FMF algorithm embedded in Astraia software (Astraia, Germany), which was updated in consecutive versions.

The results of the tests were explained to the parents. Women with a calculated risk of carrying a fetus with trisomy 21 equal to, or greater than, 1 in 100 were defined 'high risk' and had the option of an invasive procedure such as chorionic villi sampling (CVS) or amniocentesis. Also, the risk of aneuploidies was considered high if atrioventricular septal defects, holoprosencephaly, exomphalos, diaphragmatic hernia or megacystis were found on the scan. The parents were informed about the low risk of miscarriage associated with the invasive procedures. Women who wished to have an invasive test, despite their risk being considered low, were not denied this. Low risk patients were reassured that trisomies were unlikely and no further testing was offered. Together with those who refused further invasive testing, they received routine antenatal care and we followed up on the outcome of their pregnancy.

The main genetic testing that was offered was a rapid analysis of chromosomes 21, 18, 13, X and $Y$ through FISH or QF-PCR together with karyotyping. Genetic testing results were entered in the electronic database, whenever available. We performed a follow up on the outcomes of patients' pregnancies.

Patient characteristics, test results and pregnancy outcomes were obtained from the Astraia database, genetic lab records and direct questioning of the parents.

Descriptive data were presented in median and range, for continuous variables, and in numbers and percentages for categorical variables. Comparisons between categorical variables were by Fisher's exact test. We used the statistical software package SPSS 23.0 (SPSS Inc., Chicago, IL) for data analyses.

\section{RESULTS}

We screened 1358 women with singleton pregnancies at 11-13 weeks by ultrasonography, during an eight-year study period. We found a continuous increase in patients' addressability, and the number of patients scanned annually increased by $220 \%$ by the end of the study. To our knowledge, this study is one of the largest populations whose first-trimester screening data has been analysed in our area.

The ethnicity of patients was mostly Caucasian (99.9\%) and $310(22.8 \%)$ of the women were aged 35 years or older. The median maternal age was 31 years (range, 16 to 44 years) (Fig. 1). The median BMI was 22.5 (range, 14.8 to 39.1 ) and the median maternal weight was $62 \mathrm{~kg}$ (range, 38 to $114 \mathrm{~kg}$ ). The median CRL was $61 \mathrm{~mm}$ (range, 45 to $84 \mathrm{~mm}$, imposed by design). The median gestational age at the time of ultrasound evaluation was $12+3$ weeks (range, $10+5$ to $14+6$ weeks). 1259 women (92.7\%) were non-smokers. Conception was 


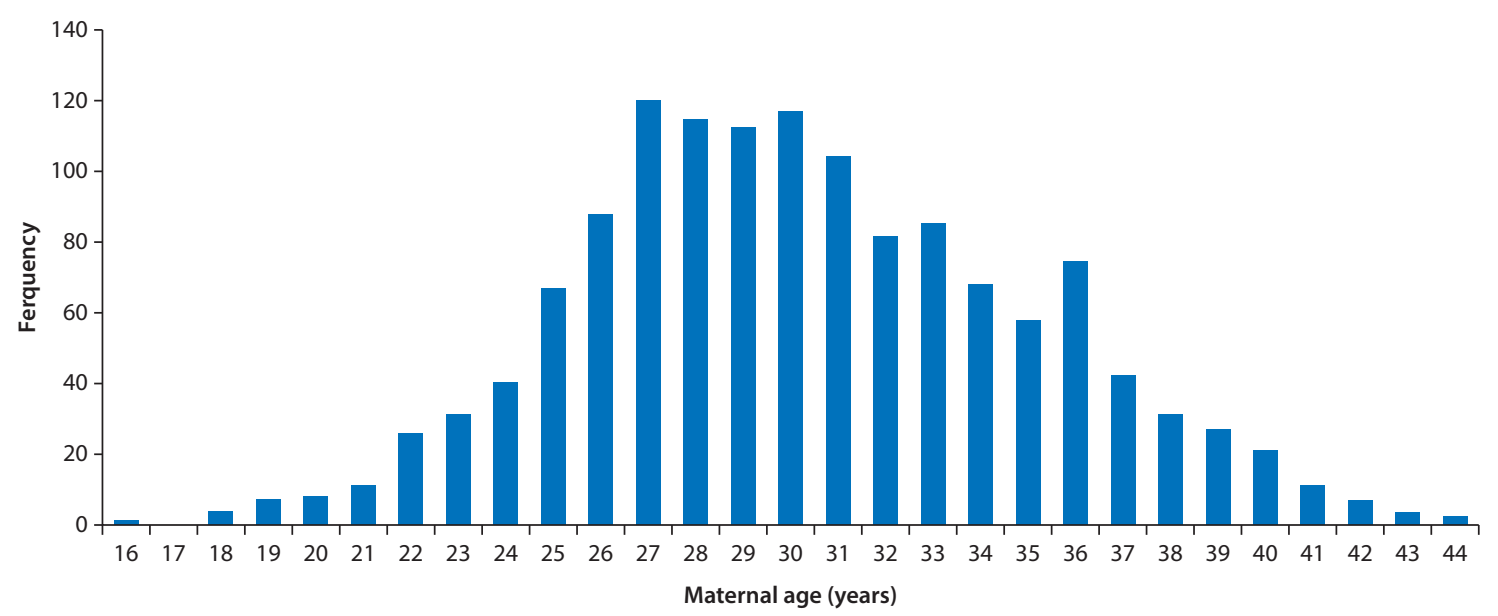

Figure 1. Maternal age distribution of the study population, in years

spontaneous in 1296 (95.4\%) of the pregnancies, by assisted reproductive technology in $53(3.9 \%)$ and not recorded in $9(0.66 \%)$. Among the 1338 pregnant women there were $18(1.3 \%)$ who had had a prior pregnancy with a chromosomal anomaly, 12 (67\%) of which had been Down syndrome.

All 1358 fetuses had an ultrasound exam with NT measurement and in 401 (29.5\%) the risk calculation was based only on ultrasound data. The most common associations of ultrasound markers for risk assessment were respectively: NT, FHR, NB, TR and DV in 1181 (87\%) cases; NT, FHR, DV and TR in 82 (6\%) cases; NT, FHR, NB and TR in 33 (2.4\%) cases; and NT, FHR, NB and DV in 21 (1.5\%) cases (Tab. 1). The fetal risk assessment using NT and all additional ultrasound markers had a relatively stable frequency during the study period, with annual values ranging from $80 \%$ to $91 \%$. Also, the association of NT measurement with at least two additional markers was done in 1317 (97\%) cases, exhibiting a stable annual frequency from $94 \%$ to $99 \%$.

The NT measurements met the FMF criteria: normal distribution of the NT deviation from the expected median for CRL measurements, $57.3 \%$ of NT values greater than the median and $6.9 \%$ greater than the $95^{\text {th }}$ percentile. The 33 fetuses (2.4\%) had a NT $>3.5 \mathrm{~mm}$ ( $99^{\text {th }}$ percentile).
In 957 (70.5\%) of the women, the trisomy 21 risk was assessed through the first trimester combined test. We found a tremendous increase in the acceptability of the combined test during the first three years of our study, with the acceptability figures stabilizing at $84-89 \%$ in the last 5 years. In 720 (75.2\%) cases, maternal blood sampling was performed at the time of, or after, the ultrasound exam, two thirds of them within two days of the ultrasound. The median gestational age at the time of the biochemistry test was 12.3 weeks (range, $9+3$ to $14+2$ weeks).

In the range of combined tests performed, the most common combination of risk calculations was based on the assessment of NT, FHR, NB, TR, DV, $\mathrm{f} \beta$-hCG and PAPP-A, namely in 851 (89\%) of cases; the next most commonly used combinations of markers was NT, FHR, DV, TR, $\mathrm{f} \beta$-hCG and PAPP-A in 50 (5.2\%) cases; followed by NT, FHR, NB, TR, f $\beta$-hCG and PAPP-A in 22 (2.3\%) cases; and NT, FHR, NB, DV, $\mathrm{f} \beta$-hCG and PAPP-A in 13 (1.4\%) cases. Overall, the association of NT measurement with at least two additional markers and biochemistry was found in 936 (97.8\%) cases (Tab. 1).

From all women with an ultrasound \pm biochemical screening in first trimester, 65 (4.8\%) had a calculated risk of trisomy 21 greater than 1 in 100, which is the screen positive

\begin{tabular}{|c|c|c|c|}
\hline & With biochemistry & No biochemistry & Total \\
\hline NT + all US markers & 851 (88.9) & $330(82.3)$ & $1181(87.0)$ \\
\hline NT + 2 US markers & $85(8.9)$ & $51(12.7)$ & $136(10.0)$ \\
\hline NT + 1 US marker & $11(1.1)$ & $16(4.0)$ & $27(2.0)$ \\
\hline NT & $10(1.0)$ & $4(1.0)$ & $14(1.0)$ \\
\hline
\end{tabular}

Data are given as n (\%); NT — nuchal translucency; US — ultrasound 


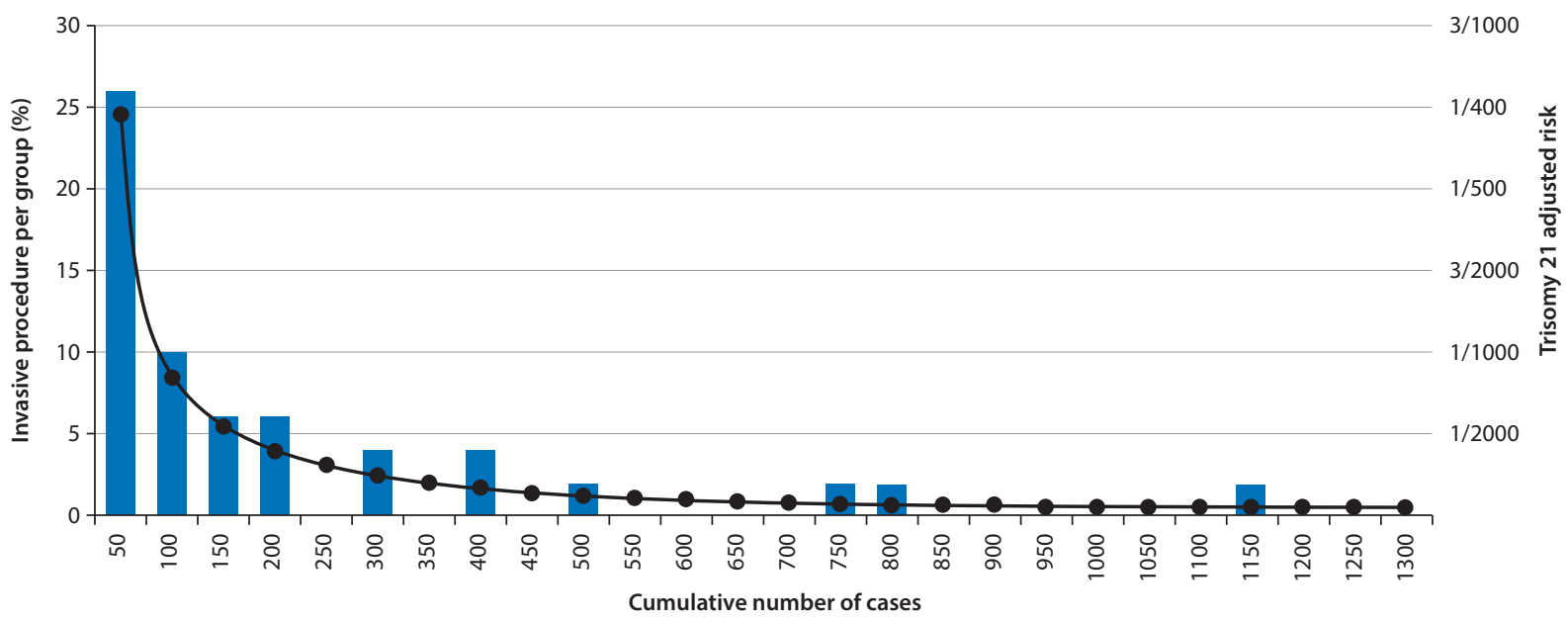

Figure 2. Frequency of invasive procedures (columns) and mean risk value (line) per groups of 50 women, ordered by trisomy 21 adjusted risk, descending. Patients with an adjuster risk for trisomy 21 and 18 greater than 1 in 100 were excluded

rate. 58 of the 65 (87.7\%) women with high risk chose to have an invasive test and the rest chose not to have any further testing. In the high-risk group, we performed 51 CVS and 10 amniocenteses. Of the 7 non-tested patients, one had an undetected trisomy 21 , three opted for pregnancy termination because of associated major fetal malformations, two were normal at birth and one removed themselves from the study. Also, some patients with a calculated risk of less than 1 in 100 elected to have invasive testing.

Overall, 92 of fetuses (6.8\%) from our first trimester population were evaluated through an invasive procedure: CVS and amniocentesis. With the low-risk patients, the closer their results were to the 1 in 100 risk margin, the more likely they were to choose to have a diagnostic procedure (Fig. 2). In the last years of our study, some women from low- or intermediate-risk groups chose to have an analysis of cell free fetal DNA of the maternal blood.

There were 24 aneuploid fetuses in our population (1.7\%): 12 cases of trisomy 21,3 cases of trisomy 18,3 cases of triploidy, 4 cases of monosomy $\mathrm{X}$, and 2 other numerical anomalies of sex chromosomes.

Based on the maternal age distribution and the age-related risk at 12 weeks' gestation, the expected number of trisomy 21 in our study population was 6 , which was not significantly different from the observed number of trisomy 21 (Fisher exact test, $p=0.165$ ). All cases with Down's syndrome had a calculated risk for trisomy 21 of between 1:2 and 1:4.

The combined test enhanced with ultrasound markers detected 11 of the 12 cases of trisomy 21 , with a first trimester detection rate (DR) of $91.7 \%$. The missed trisomy 21 was one of our first series' cases and the abnormal ductus venous flow was not recognized. Then, at 30 weeks the fetal scan demonstrated growth restriction and several anomalies. The final diagnosis was made at birth.
39 fetuses (2.8\%) had these types of structural anomalies: ventriculomegaly, holoprosencephaly, acrania, cleft lip/palate, diaphragmatic hernia, gastroschisis, omphalocele, hydrops, megacystis, and limb abnormalities. Overall, we found $1.2 \%$ (17 cases) of the fetuses had major heart defects.

\section{DISCUSSION}

In this paper we present the population characteristics and the results from our implementation of the combined test enhanced with additional ultrasound markers, at 11 to $13+6$ weeks' in a private center. Overall, $87 \%$ of patients in the study had an evaluation of all additional markers (NB, DV, TR) and in 97\% at least two markers were assessed, in any combination. $70 \%$ of patients were also evaluated using maternal serum biochemistry. In our population, the detection rate for trisomy 21 was high, at $91.7 \%$ with a FPR of $4.8 \%$.

When comparing the standard combined test, our results were found to be similar to both those from the most recent prospective multicenter study of Nicolaides and col. [8] (92\% DR for a FPR of 4.6\%), and the original model study [5] (90\% DR for a FPR of 3\%). Two other recent studies also reported similar detection rates $[18,19]$ of $91.6 \%$ and $93.75 \%$, respectively.

When we compare our results with those from studies that used a combined test enhanced by all additional ultrasound markers, we achieved the same detection rate as that published by Karadzov-Orlic et al. [20] (93\% DR for FPR of $4.8 \%)$, at a cutoff of 1:275. However, we failed to reach the excellent $100 \%$ detection rates obtained by Hsiao et al. [14] or Ghaffari et al. [15] at lower FPRs of $2.4 \%$ or $3.4 \%$, respectively.

Our results were at the same DR, but with a lower FPR than those found by studies which used an ultrasound-based protocol (NT and all additional markers). Thus, at a 5\% FPR, Abele et al. [13] reached a DR for T21 of $97.5 \%$, while Wiechec et al. [11, 12] achieved a DR of $94-95 \%$. 
Finally, ourT21 detection rate was much higher than that of the triple test, which has been found to be around $70 \%$, and higher than that of the quadruple test, which has an overall detection rate of approximately $80 \%[21,22]$. When compared with second trimester maternal serum tests, the first trimester combined test achieves a better detection rate and provides an earlier diagnosis. Therefore, we demonstrated the usefulness of the first trimester combined test in Down syndrome screening.

Our median maternal age was 31 years, which was comparable to other values registered in Spain (32.4 years in 2016) [23], Romania (29.6 years in 2017) [18, 24] or in Poland (34 years in 2017) [19], for first-trimester screening population.

Our patients were quite slim, with a median BMI of 22.5, which favored the incorporation of all additional first trimester ultrasound markers into the screening. Overall, we incorporated at least two additional markers in the screening of $97 \%$ of patients.

We found an increased acceptability of the combined test instead versus ultrasound first-trimester screening only, especially in the last 5 years of the study, when $84-98 \%$ of the patients accepted maternal serum screening. This is remarkable, in conditions when all screening investigations undertaken at the patients' own cost. However, we acknowledge that the biochemical component of the combined test has only recently been covered by public health insurance (namely, from 2015, the last but one year of the study), and that the FMF-certified biochemistry machines were either not available in the first years or the costs were high; so, many patients preferred not to pay directly for serum testing and others were not particularly interested in a more accurate risk assessment for aneuploidies.

Eighty eight percent of the women with positive screening results opted to have further invasive diagnostic testing. No miscarriages were reported. However, other patients from both the intermediate- and low-risk groups also elected to have the invasive procedure. With the low-risk patients, the closer their results were to the 1 in 100 risk margin, the more likely they were to choose to have a diagnostic procedure (Fig. 2). Thus, at a mean adjusted risk for trisomy 21 of 1 in $400,25 \%$ of the women chose to have a diagnostic procedure. The percentage dropped exponentially, so, at 1 in 1000, only $10 \%$ elected to have an invasive test. These figures could in the future be lowered further through the availability of cell free fetal DNA analysis of the maternal blood [25].

The small sample size of our study, in comparison with larger prospective studies, could be a weakness, and associated with the small number of T21 cases in our study, could have lowered the detection rate. In addition, because the study was conducted in a single private center, the result cannot fully represent the screening performance across our whole population, due to the possibility of selection bias. Moreover, the overall screening performance might be affected by the test timing [26]. Also, the study was limited with regard to the diagnosis of other chromosomal anomalies, because the main genetic test was a rapid analysis of chromosomes 21, 18, 13, $X$ and $Y$ through FISH or QF-PCR. The test was offered free of charge in the final 4 years of our study as the costs were reimbursed by health assurance. The frequency of karyotyping prior to this, was therefore low, because of the costs.

\section{CONCLUSIONS}

The purpose of our study was to provide information on the screening performance of first-trimester combined testing and on the population characteristics in a private medical center. We compared the results with those of Western countries and with different screening methodologies.

The detection rate and FPR of the first trimester screening conducted in our medical center were comparable with others that had been reported previously. A combined test enhanced with all additional ultrasound markers did not show any substantial improvement in T21 detection rates compared with using only one of the additional markers as recommended by FMF. In our conditions, this early screening method can improve detection rates without increasing invasive procedures and can detect some early major fetal anomalies. Finally, our data shows that the combined first trimester test for aneuploidies is a robust test, providing an effective and reliable screening.

\section{REFERENCES}

1. Kagan KO, Wright $D$, Baker A, et al. Screening for trisomy 21 by maternal age, fetal nuchal translucency thickness, free beta-human chorionic gonadotropin and pregnancy-associated plasma protein-A. Ultrasound in Obstetrics and Gynecology. 2008; 31(6): 618-624, doi: 10.1002/uog.5331.

2. Cicero $\mathrm{S}$, Rembouskos $\mathrm{G}$, Vandecruys $\mathrm{H}$, et al. Likelihood ratio for trisomy 21 in fetuses with absent nasal bone at the 11-14-week scan. Ultrasound Obstet Gynecol. 2004; 23(3): 218-223, doi: 10.1002/uog.992, indexed in Pubmed: 15027007

3. Kagan $\mathrm{KO}$, Valencia $\mathrm{C}$, Livanos $\mathrm{P}$, et al. Tricuspid regurgitation in screening for trisomies 21,18 and 13 and Turner syndrome at $11+0$ to $13+6$ weeks of gestation. Ultrasound Obstet Gynecol. 2009; 33(1): 18-22, doi: 10.1002/uog.6264, indexed in Pubmed: 19031473.

4. Maiz N, Valencia C, Kagan KO, et al. Ductus venosus Doppler in screening for trisomies 21, 18 and 13 and Turner syndrome at 11-13 weeks of gestation. Ultrasound Obstet Gynecol. 2009; 33(5): 512-517, doi: 10.1002/uog.6330, indexed in Pubmed: 19338027.

5. Kagan KO, Wright D, Valencia C, et al. Screening for trisomies 21,18 and 13 by maternal age, fetal nuchal translucency, fetal heart rate, free beta-hCG and pregnancy-associated plasma protein-A. Hum Reprod. 2008; 23(9): 1968-1975, doi: 10.1093/humrep/den224, indexed in Pubmed: 18544579.

6. Nicolaides K. Screening for fetal aneuploidies at 11 to 13 weeks. Prenatal Diagnosis. 2011; 31(1): 7-15, doi: 10.1002/pd.2637.

7. Kagan KO, Etchegaray A, Zhou Y, et al. Prospective validation of first-trimester combined screening for trisomy 21. Ultrasound Obstet Gynecol. 2009; 34(1): 14-18, doi: 10.1002/uog.6412, indexed in Pubmed: 19526452.

8. Santorum M, Wright $D$, Syngelaki A, et al. Accuracy of first-trimester combined test in screening for trisomies 21,18 and 13. Ultrasound Obstet Gynecol. 2017; 49(6): 714-720, doi: 10.1002/uog.17283, indexed in Pubmed: 27549925

9. Wright $D$, Syngelaki $A$, Bradbury I, et al. First-trimester screening for trisomies 21,18 and 13 by ultrasound and biochemical testing. Fetal 
Diagn Ther. 2014; 35(2): 118-126, doi: 10.1159/000357430, indexed in Pubmed: 24356462.

10. Maiz $\mathrm{N}$, Wright $\mathrm{D}$, Ferreira $A F$, et al. A mixture model of ductus venosus pulsatility index in screening for aneuploidies at 11-13 weeks' gestation. Fetal Diagn Ther. 2012; 31(4): 221-229, doi: 10.1159/000337322, indexed in Pubmed: 22614037.

11. Wiechec M, Nocun A, Knafel A, et al. Combined screening test for trisomy 21 - is it as efficient as we believe? J Perinat Med. 2017; 45(2): 185-191, doi: 10.1515/jpm-2016-0031, indexed in Pubmed: 27008689.

12. Wiechec M, Knafel A, Nocun A, et al. How Effective Is First-Trimester Screening for Trisomy 21 Based on Ultrasound Only? Fetal Diagn Ther. 2016; 39(2): 105-112, doi: 10.1159/000434632, indexed in Pubmed: 26303666.

13. Abele $H$, Wagner $P$, Sonek J, et al. First trimester ultrasound screening for Down syndrome based on maternal age, fetal nuchal translucency and different combinations of the additional markers nasal bone, tricuspid and ductus venosus flow. Prenat Diagn. 2015; 35(12): 1182-1186, doi: 10.1002/pd.4664, indexed in Pubmed: 26223508.

14. Hsiao $\mathrm{CH}$, Cheng PJ, Shaw SW, et al. Extended first-trimester screening using multiple sonographic markers and maternal serum biochemistry: a five-year prospective study. Fetal Diagn Ther. 2014; 35(4): 296-301, doi: 10.1159/000357564, indexed in Pubmed: 24503519.

15. Ghaffari SR, Tahmasebpour AR, Jamal A, et al. First-trimester screening for chromosomal abnormalities by integrated application of nuchal translucency, nasal bone, tricuspid regurgitation and ductus venosus flow combined with maternal serum free $\beta$-hCG and PAPP-A: a 5 -year prospective study. Ultrasound Obstet Gynecol. 2012; 39(5): 528-534, doi: 10.1002/uog.10051, indexed in Pubmed: 21793085.

16. Iliescu D, Tudorache S, Comanescu A, et al. Improved detection rate of structural abnormalities in the first trimester using an extended examination protocol. Ultrasound Obstet Gynecol. 2013; 42(3): 300-309, doi: 10.1002/uog.12489, indexed in Pubmed: 23595897.

17. Nemescu D, Berescu A, Rotariu C. Variation of safety indices during in the learning curve for color Doppler assessment of the fetal heart at $11+0$ to $13+6$ weeks' gestation. Med Ultrason. $2015 ; 17(4)$ : 464-474, doi: 10.11152/mu.2013.2066.174.vsy, indexed in Pubmed: 26649341.
18. Veduta A, Vayna AM, Duta S, et al. The first trimester combined test for aneuploidies - a single center experience. J Matern Fetal Neonatal Med. 2017 [Epub ahead of print]: 1-6, doi: 10.1080/14767058.2017.1336220, indexed in Pubmed: 28553771.

19. Czuba B, Zarotyński D, Dubiel M, et al. Screening for trisomy 21 based on maternal age, nuchal translucency measurement, first trimester biochemistry and quantitative and qualitative assessment of the flow in the DV - the assessment of efficacy. Ginekol Pol. 2017; 88(9): 481-485, doi: 10.5603/GP.a2017.0088, indexed in Pubmed: 29057433.

20. Karadzov-Orlic N, Egic A, Filimonovic D, et al. Screening performances of abnormal first-trimester ductus venosus blood flow and increased nuchal translucency thickness in detection of major heart defects. Prenat Diagn. 2015; 35(13): 1308-1315, doi: 10.1002/pd.4693, indexed in Pubmed: 26408089.

21. ACOG Committee on Practice Bulletins. ACOG Practice Bulletin No. 77: screening for fetal chromosomal abnormalities. Obstet Gynecol. 2007; 109(1): 217-227, doi: 10.1097/00006250-200701000-00054, indexed in Pubmed: 17197615.

22. Carabineanu A, Navolan D, Birsasteanu F, et al. The Effect of Chemical Compounds from Cigarettes Smoke on First Trimester Biochemical Markers. Revista De Chimie. 2017; 68(9): 2122-2124.

23. Gil MM, Brik M, Casanova C, et al. Screening for trisomies 21 and 18 in a Spanish public hospital: from the combined test to the cell-free DNA test. J Matern Fetal Neonatal Med. 2017; 30(20): 2476-2482, doi: 10.1080/14767058.2016.1253062, indexed in Pubmed: 27806655.

24. Navolan D, Vladareanu S, Ciohat I, et al. Distribution of Biochemical and Ultrasound Markers Values in the First Trimester Screening Program in Timisoara. Revista De Chimie. 2017; 68(7): 1636-1639.

25. Radoi VE, Bohiltea $\mathrm{CL}$, Bohiltea RE, et al. Cell free fetal DNA testing in maternal blood of Romanian pregnant women. Iran J Reprod Med. 2015; 13(10): 623-626, indexed in Pubmed: 26644790.

26. Gyselaers WJA, Vereecken AJ, Van HerckEJH, et al. Population screening for fetal trisomy 21: easy access to screening should be balanced against a uniform ultrasound protocol. Prenat Diagn. 2005; 25(11): 984-990, doi: 10.1002/pd.1217, indexed in Pubmed: 16240322. 